\title{
Norois
}

Environnement, aménagement, société

\section{La spatialité des conflits d'usage au sein des zones périurbaines en Île-de-France : analyse empirique d'une modalité peu connue de la gouvernance des territoires}

The spatiality of land use conflicts in the Île-de-France periurban areas: a way of analyse one side of the territory local governance

\section{Ségolène Darly}

\section{(2) OpenEdition}

Journals

Édition électronique

URL : https://journals.openedition.org/norois/2691

DOI : 10.4000/norois.2691

ISBN : 978-2-7535-1557-4

ISSN : 1760-8546

Éditeur

Presses universitaires de Rennes

Édition imprimée

Date de publication : 15 décembre 2008

Pagination : 127-146

ISBN : 978-2-7535-0805-7

ISSN : 0029-182X

Référence électronique

Ségolène Darly, "La spatialité des conflits d'usage au sein des zones périurbaines en Île-de-France analyse empirique d'une modalité peu connue de la gouvernance des territoires », Norois [En ligne], 209 | 2008/4, mis en ligne le 01 mars 2011, consulté le 14 janvier 2022. URL : http:// journals.openedition.org/norois/2691; DOI : https://doi.org/10.4000/norois.2691 


\title{
LA SPATIALITÉ DES CONFLITS D'USAGE AU SEIN DES ZONES PÉRIURBAINES EN ÎLE-DE-FranCE : ANALYSE EMPIRIQUE D'UNE MODALITÉ PEU CONNUE DE LA GOUVERNANCE DES TERRITOIRES
}

\author{
SÉGOLÈNE DARLY \\ INRA UMR SADAPT, équipe Proximités \\ 16 rue Claude-Bernard - 75005 PARIS \\ darly@agroparistech.fr
}

\section{RÉSUMÉ}

Cet article part du principe que la gouvernance des territoires périurbains est marquée par des processus conflictuels, qui y sont particulièrement vifs en raison de la diversité et de la proximité d'usages antagoniques de l'espace. La différenciation de la gouvernance territoriale en Île-de-France, que nous cherchons à mettre en évidence, va donc être analysée ici à partir d'une géographie des conflits. Pour cela, nous avons constitué un corpus de conflits à partir de la consultation de la presse quotidienne régionale sur une année, en recherchant les spécificités de ces conflits au sein des sept territoires périurbains franciliens, hérités des anciens pays ruraux. Les caractéristiques spécifiques des territoires périurbains en matière de conflits sont analysées à partir d'une grille de lecture de la spatialité des conflits organisée en trois étapes: celle de la géographie des objets et des ressources mis en jeu, celle de l'inscription territoriale des acteurs en conflit, et enfin, celle des productions spatiales des conflits. Cette analyse met en évidence trois figures territoriales de la conflictualité qui participent à la différenciation des modalités de la gouvernance : celle des territoires sous forte pression environnementale, celle des territoires à forte résistance identitaire, et celle des territoires de conflictualité occasionnelle.

MoTS CLÉ : conflits d'usage-gouvernance-géographie - presse quotidienne régionale - territoires périurbains - Île-de-France

\footnotetext{
ABSTRACT

The spatiality of land use conflicts in the île-de-France periurban areas : a way of analyse one side of the territory local governance

This paper is inspired by the fact that territorial governance, in periurban areas, is dependant of conflictual antagonisms. The territorial differentiation of governance in the Ille-de-France periurban area can therefore be analysed thanks to conflicts geography. This method is applied to seven periurban territories of the Ille-de-France region, using a set of conflictual situations gathered from the reading of the daily regional news paper. The specific characteristics of the conflicts within each territory are identified following three steps: the geography of the objects and resources that constitute the stake of the conflict, the territorial identity of the actors, and the geography of the spaces produced by the conflict. This analysis shows the differences of conflictuality between three types of territories: territories
} 
under great environmental pressure, territories with identity based resistance capacities, and territories of occasional conflictuality.

KEY WORDS : land use conflicts - governance - geography - regional daily paper-periurban territories - Ille-de-France

\section{Introduction}

\section{Prise D’autonomie des ZONES PÉRIURBAINES ET DIFFÉRENCIATION TERRITORIALE DES MODALITÉS DE LA GOUVERNANCE LOCALE}

L'agglomération parisienne étend son aire d'influence sur l'ensemble des territoires de la région Île-de-France, jusqu'à s'affranchir de ses frontières régionales pour toucher les départements limitrophes (fig. 1). La dernière typologie INSEE des communes franciliennes enregistre ainsi la quasi disparition des communes strictement rurales, ainsi que le développement interdépendant de deux zones : la zone de l'urbain dense, qui s'étend en tâche d'huile depuis le centre de Paris, et une vaste couronne périurbaine, qui constitue $85 \%$ de la superficie régionale et accueille $22 \%$ de ses habitants. Amorcée dès la fin des années 1960, et achevée en ce début de XXI ${ }^{\mathrm{e}}$ siècle, la périurbanisation progressive de l'ensemble des territoires locaux de la région est un phénomène unique, d'une ampleur, et d'une antériorité sans égale par rapport aux autres « couronnes périurbaines » françaises ${ }^{1}$.

Cet état n'est cependant pas que le résultat de l'avancée d'un front urbain uni et coordonné qui entraînerait la disparition brutales des espaces ouverts (agricoles, forestiers et naturels) au profit de zones entièrement occupées par du bâti, mais bien plus la conséquence d'une polarisation des emplois et des activités de résidents ruraux vers les pôles urbains de l'agglomération centrale. Ainsi, en termes de morphologie de l'espace, les limites de la zone urbaine dense centrale ont faiblement évolué depuis les années 1980, alors que des territoires proches et éloignés ont accueilli de plus en plus de résidents, tout en conservant une morphologie rurale ou moyennement bâtie ${ }^{2}$. Cet élément sert d'ailleurs bien souvent, pour ces communautés locales, de critère de différenciation identitaire vis-à-vis de l'agglomération centrale. Ce phénomène oblige les observateurs de la gouvernance des territoires périurbains à envisager un retournement des dynamiques locales, à l'instar de M.-C. Jaillet, qui ne seraient plus seulement marquées par une mise en pratique des politiques régionales ou métropolitaines qui préparent l'arrivée de la ville, mais bien par une tendance à l'autonomisation des dispositifs locaux d'organisation, portée par des communautés périurbaines renouvelées (Jaillet, 2004). Cette hypothèse implique que la gouvernance de ces territoires ne peut être réduite aux modalités de pilotage du gouvernement urbain (État et Région dans notre cas), mais doit aussi prendre en compte le rôle déterminant des dynamiques locales de gouvernance dans la différenciation des territoires périurbains (Bryant, 1995).

\section{Une APPROCHE DE LA GOUVERNANCE PAR LES PROCESSUS CONFLICTUELS}

L'analyse de ces dynamiques locales de gouvernance est généralement abordée selon trois angles : celui de la territorialisation des politiques publiques (Bertrand et Tobron, 2002), celui

1. Ce constat avait été fait dans les années 1980 par P. Cadène (1990), qui avait renoncé à inclure la région parisienne dans son analyse comparative de la conflictualité des couronnes périurbaines françaises.

2. L'IAURIF établit une typologie des communes de l'T̂le-de-France en fonction de critères classiques de démographie mais aussi en fonction de la morphologie de l'espace. Cette typologie distingue ainsi des communes à la morphologie urbaine, des communes à la morphologie périurbaine, et des communes (périurbaines) à la morphologie rurale (Iaurif, 2004). 


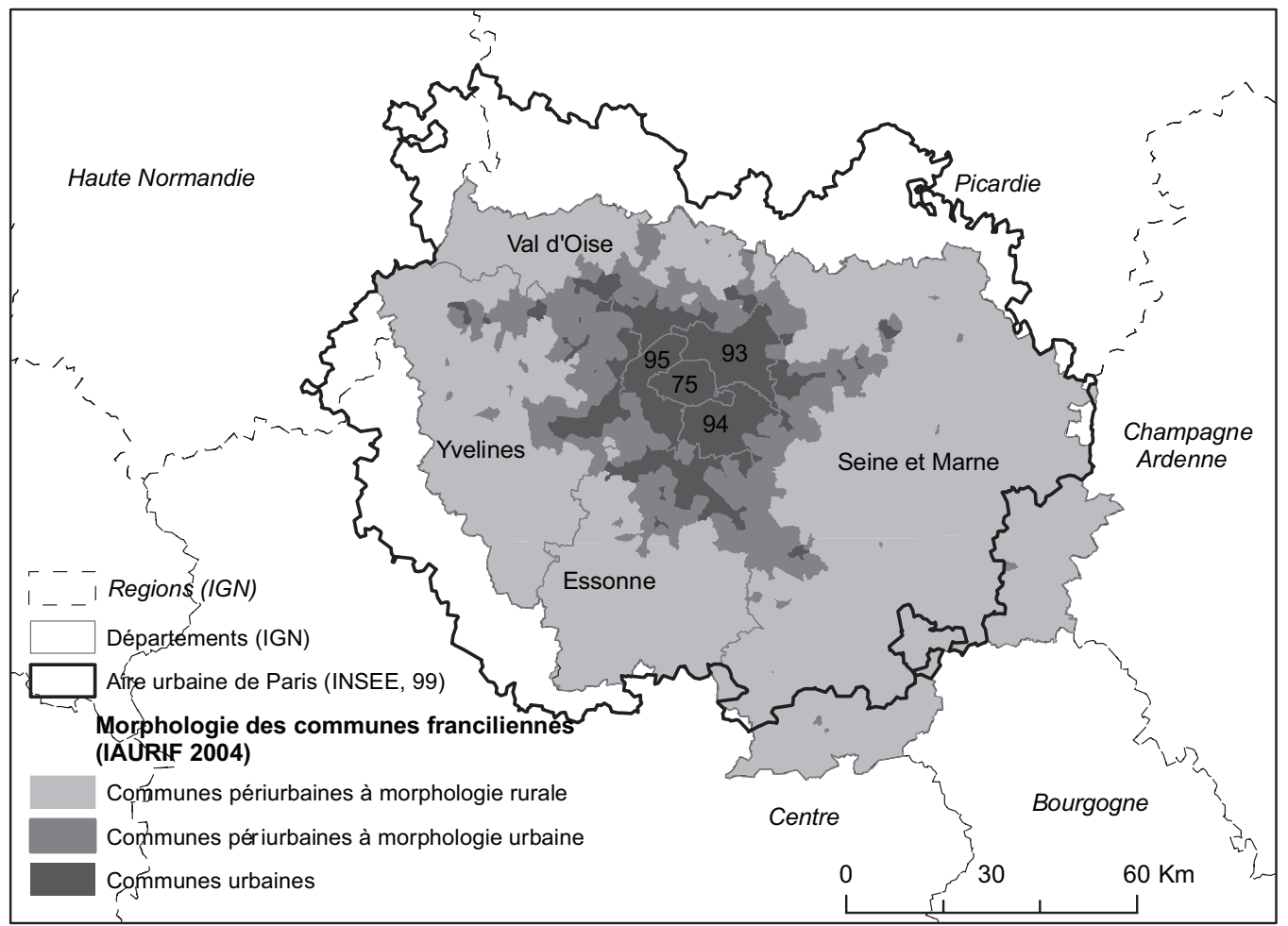

Figure 1 : Localisation des limites administratives de l'Île-de-France et des limites de l'aire urbaine de Paris (Sources : INSEE 99, IAURIF, 2003) ropolitan area.

Administrative boundaries of Île-de-France, compared with the functional boundaries of Paris met-

de la coopération autour de projets locaux innovants (Papy et Torre, 2002), mais aussi, est c'est à ce dernier que nous nous intéressons dans cette communication, celui de la régulation sociale (Reynault, 1997), par le conflit, des usages, des activités et des comportements à l'intérieur des territoires. Adoptant une posture résolument tournée vers l'action, la majeure partie des travaux de géographie propose en effet une analyse de l'inscription spatiale des dynamiques coopératives, productrices d'outils de gouvernance. En comparaison, l'analyse empirique des dynamiques conflictuelles n'émerge que plus récemment à travers plusieurs programmes de recherches complémentaires $^{3}$. En posant l'hypothèse que les modes de coordinations entre acteurs sont autant conflictuels que coopératifs, c'est une grande partie des modalités de la gouvernance locale qui reste encore à mettre en évidence (Kirat et Torre, 2006).

Cet article propose donc de contribuer à l'analyse des dynamiques territoriales de la gouvernance en périurbain, en s’intéressant aux processus conflictuels localisés.

Le choix de cet angle d'analyse n'est pas totalement indépendant des caractéristiques de l'̂lle-deFrance. L'espace régional y est en effet l'objet d'une compétition exacerbée entre les acteurs des différents secteurs secondaires (bâtiment, construction, transformation) et tertiaires (logistique

3. Le programme « Conflits d'usage dans les territoires ruraux et périurbains » de l'équipe Proximités du SADAPT, mais aussi le séminaire «Politiques publiques et Territoires » du Laboratoire Ville, Société Territoire de l'Université de Tours, plus spécifiquement dédié à la question des conflits, et enfin, le programme sur l’analyse spatiale des conflits urbains porté par le Centre de Recherche pour l'Aménagement Durable, de l'Université de Laval, au Québec. 
et commerce) qui cherchent la proximité des services de l'agglomération parisienne mais qui sont aussi nécessaires à son fonctionnement (gestion des déchets, services publics, logements individuels ou collectifs). À ces usages en concurrence s'ajoutent par ailleurs les activités du secteur primaire, carriers, producteurs et fournisseurs d'énergie (dont éoliennes depuis la période récente), et bien sûr les activités forestières, cynégétiques et agricoles, qui valorisent à elles seules près de $80 \%$ de l'espace régional. La proximité de toutes ces activités, partiellement antagoniques, dans un territoire restreint hébergeant douze millions d'individus, dont la mobilité résidentielle est de plus en plus difficile, rassemble les conditions d'une multitude de tensions et de conflits entre les différents usagers de l'espace et entre les différentes échelles de gestion. De plus, la situation de région-capitale de l'Île-de-France implique que, aux côtés des collectivités locales chargées de la gestion de l'espace et de l'aménagement, l'État soit lui-même aménageur d'une partie du territoire et cherche à préserver le rôle central, voire centralisateur, des infrastructures métropolitaines. Les contradictions entre intérêt national, régional, local n'en sont que plus fortes et sont autant de sujets potentiels de conflit.

Si les territoires périurbains franciliens semblent donc être propices à l'émergence de processus conflictuels variés, la littérature francophone sur la géographie des conflits d'usage des espaces périurbains est assez restreinte et se fonde en particulier sur les travaux de plusieurs recherches doctorales. Tous se basent, plus ou moins explicitement, sur les hypothèses d'un courant de la sociologie qui réfute la nature dysfonctionnelle des conflits. Plusieurs travaux fondateurs ont ainsi introduit la notion du conflit comme processus social (Touraine, 1993). La géographie de l'action s'en est saisie, notamment pour l'étude des reconfigurations des espaces périurbains, dont la nature multifonctionnelle est à la base d'une forte conflictualité (Donadieu et Fleury, 2003).

À la fin des années 1970, cette géographie s'est d'abord intéressée à la confrontation urbain - rural dans les territoires périphériques des villes, dont l'ampleur spatiale prend une dimension incontournable. Le travail de P. Cadène (1990), à partir d'enquêtes de terrain, est mené autour de dix villes françaises, qui voient apparaître au début des années 1980 le phénomène de formation des couronnes périurbaines.

Une quinzaine d'années plus tard, la révolution environnementaliste, née aux États-Unis, s'est développée en France et les conflits pour la protection de la nature et des ressources écologiques se multiplient, en réponse aux périodes de modernisation et d'extension des infrastructures routières, énergétiques et de loisirs de masse (urbanisation des zones de montagne et littorales). C'est B. Charlier (1999) qui caractérise géographiquement ce phénomène à partir des événements conflictuels recensés par la revue Combat Nature. Cette étude fera date, et est encore aujourd'hui le seul indicateur de conflictualité déterminé à l'échelle nationale (Guérin, 2005).

Par la suite, les travaux de plusieurs aménagistes (Dziedzicki, 2001, Faburel, 2001, Lecourt, 2003) analysent les conditions d'émergence et d'extension de la contestation des infrastructures, en prenant pour principaux objets d'étude les acteurs associatifs et leurs impacts sur les modes de gouvernance de l'espace.

Parallèlement, depuis le début des années 2000, l'émergence du concept de développement durable ramène sur le devant de la scène la nécessité d'une gestion intégrée des ressources et le rôle central des modes de pilotage des conflits d'usage des ressources partagées (Beuret, 2006). La question des interactions entre acteurs, qui avait pourtant été évoquée dès la thèse de Cadène, resurgit et donnera lieu à plusieurs travaux issus de la discipline des sciences de gestion (Michel, 2003). La géographie se saisit aussi de la question des processus sociaux territorialisés et tente de caractériser l'inscription spatiale des différentes figures des interactions conflictuelles. Les travaux d’A. Cadoret (2006) sur les réseaux d'acteurs liés aux conflits sur le littoral languedocien illustrent ce nouveau champ de développement.

On notera par ailleurs, que le lien entre conflits et gouvernance fait aussi l'objet d'une attention particulière de la part des géographes de l'urbain. Ainsi, les travaux de l'université de Tours (Melé, 2004), ainsi que de l'Université de Laval au Québec (Joérin et al., 2005), s’intéressent aux conflits 
dans la ville et son aire urbaine. Ils tiennent plus particulièrement compte de la territorialisation des politiques urbaines de régulation, objet d'étude privilégié de cette spécialité.

On devine donc que la gouvernance liée aux conflits d'usage des espaces périurbains devient aujourd'hui un objet qui autorise emprunts et synergies entre géographie urbaine, aménagement et géographie rurale.

\section{DES TERRITOIRES DE DIFFÉRENCIATION HERITÉS DE L’HISTOIRE RÉGIONALE DES PAYS RURAUX}

Pour tester l'existence de processus conflictuels territorialisés, nous nous baserons sur un découpage de l'espace régional en sept pays ruraux $^{4}$ (fig. 2). Sans existence institutionnelle reconnue, ce découpage a été retenu à la suite d'entretiens auprès d'acteurs de la gestion des territoires, qui se réfèrent souvent à cet héritage identitaire pour illustrer les différences de réactivités des populations locales.

Les sept territoires ainsi identifiés décrivent une diversité de situations, qui rend compte des disparités spatiales et socio-économiques persistantes auxquelles se trouve confronté le gouvernement régional (tableau 1). Le plus vaste est celui de la Brie (Brie française et Brie champenoise), qui est aussi celui dont la population a le plus augmenté entre 1990 et 1999. Le plus peuplé reste cependant celui de la Ceinture de Paris, qui correspond aux terroirs de la zone de confluence des cours d'eau franciliens, et aux communes densément peuplées de la proche couronne parisienne. Les territoires les plus «agricoles » sont ceux des vastes plateaux céréaliers du Hurepoix et du Vexin, avec plus de $65 \%$ d'espaces cultivés, alors que les plus «forestiers » sont les territoires du Gâtinais (massif de Fontainebleau) et du Mantois (massif de Rambouillet) avec plus de 30 \% d'espaces boisés. Enfin, le Mantois et le Hurepoix au sud-ouest, anciennes zones d'accueil des premières grandes vagues de périurbanisation par l'habitat individuel, sont caractérisés aujourd'hui par une population plus « aisée » au regard du revenu moyen annuel par foyer fiscal.

\begin{tabular}{|l|c|c|c|c|c|c|}
\hline & $\begin{array}{c}\text { Superficie } \\
\left(\mathrm{km}^{2}\right) \\
\text { INSEE, 99 }\end{array}$ & $\begin{array}{c}\text { Pop. (1 000) } \\
\text { INSEE, 99 }\end{array}$ & $\begin{array}{c}\text { Var. pop. } \\
\text { (INSEE, 90- } \\
99)\end{array}$ & $\begin{array}{c}\text { Sup. agrico- } \\
\text { les (IAURIF } \\
2003)\end{array}$ & $\begin{array}{c}\text { Sup. forêts } \\
\text { (IAURIF } \\
\text { 2003) }\end{array}$ & $\begin{array}{c}\text { Revenus } \\
\text { annuels moyen } \\
\text { des ménages } \\
\text { (DGI 2006) }\end{array}$ \\
\hline $\begin{array}{l}\text { Ceinture de } \\
\text { Paris }\end{array}$ & 1433 & $\mathbf{4 7 5 0}$ & $1,8 \%$ & $15,4 \%$ & $16,6 \%$ & 19711 \\
\hline Valois & $\mathbf{7 4 8}$ & 337 & $8,1 \%$ & $61,8 \%$ & $13 \%$ & 18730 \\
\hline Brie & $\mathbf{4 3 0 3}$ & 990 & $\mathbf{1 2 , 4} \%$ & $63,6 \%$ & $21,2 \%$ & 18767 \\
\hline Gatinais & 1732 & 299 & $5,7 \%$ & $53,1 \%$ & $\mathbf{3 5} \%$ & 19627 \\
\hline Hurepoix & 1141 & 295 & $7 \%$ & $\mathbf{6 6} \%$ & $19,6 \%$ & 22450 \\
\hline Mantois & 1828 & 771 & $4,8 \%$ & $47,5 \%$ & $\mathbf{3 2} \%$ & $\mathbf{2 3 4 4 0}$ \\
\hline Vexin & 635 & 83 & $\mathbf{1 2 , 2} \%$ & $\mathbf{6 8 , 6} \%$ & $20 \%$ & 21381 \\
\hline
\end{tabular}

Tableau 1 : Principales caractéristiques des territoires périurbains issus des anciens pays ruraux, en Île-de-France Statistical caracteristics of the " rural countries » of the Île-de-France

À partir d'un recensement des conflits d'usage des espaces périurbains en Île-de-France, nous proposons une grille de lecture des caractéristiques des conflits, spécifiques à chacun de ces ter-

4. Le découpage des territoires franciliens proposé est issu d'une reconstitution des anciens pays d'Île-de-France de l'Ancien Régime, abolis après la Révolution, mais dont la relative unité reste perceptible aujourd'hui. Chaque territoire rural a été reconstitué en agrégeant les petites régions agricoles (Agreste) qui nous semblent être héritées de ces anciens pays. (Iaurif, 2004). 


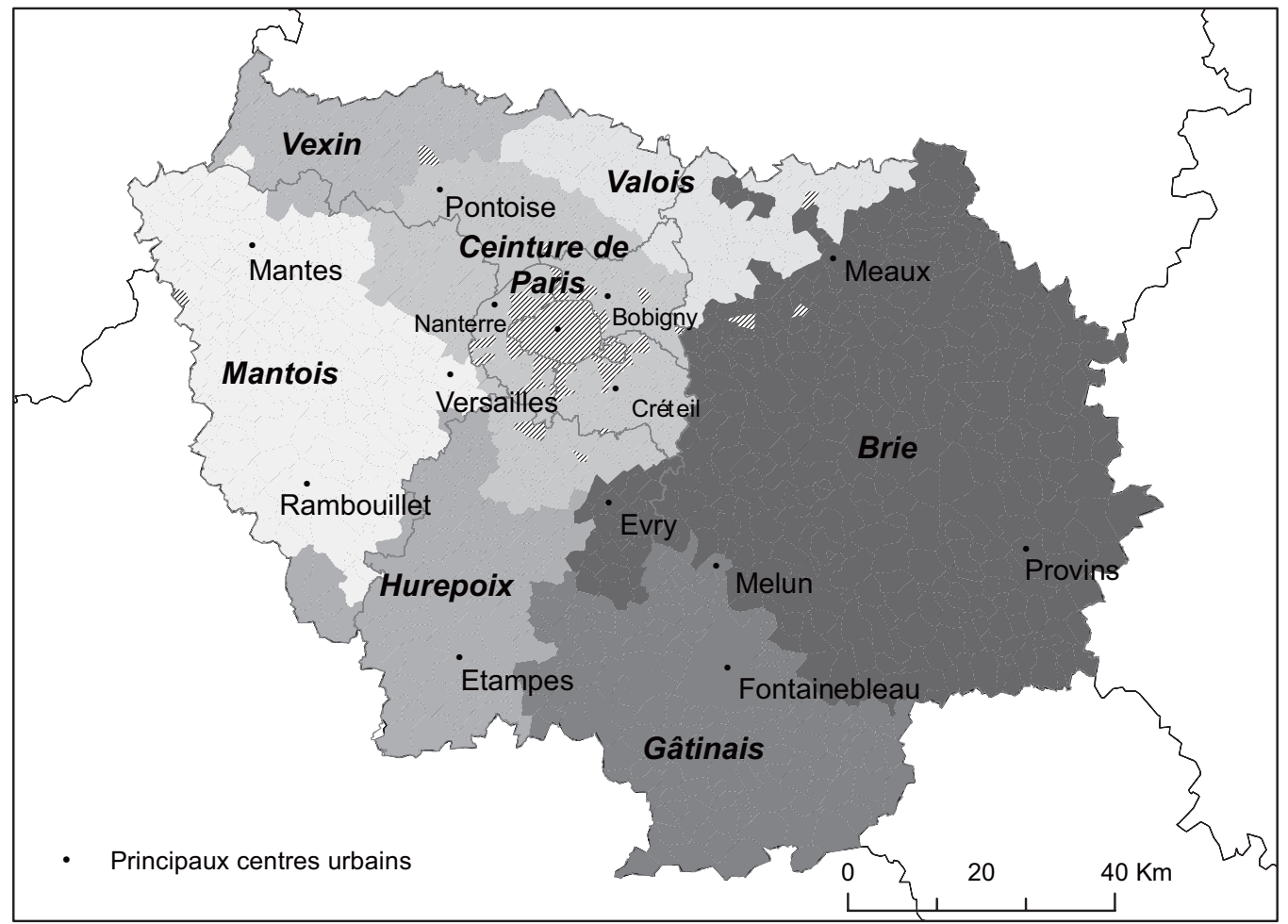

Figure 2 : Localisation des principales villes de la zone rurale et reconstitution des territoires ruraux issus des pays de l'Ancien Régime (Source : Darly 2008, d'après IAURIF, 2004) of Île-de-France.

Major local cities and hypothetic boundaries of "rural countries" drawn from the historical "pays"

ritoires. Elle articule trois domaines d'analyse de la spatialité des conflits : le domaine des espaces du conflit et des enjeux territoriaux qu'ils révèlent, celui des groupes d'acteurs et de leur inscription spatiale, et enfin, celui des productions spatiales des conflits. Après une brève présentation de la méthode de recensement utilisée et une première description synthétique du corpus de conflits, nous détaillerons successivement les résultats relatifs aux trois dimensions de la spatialité des conflits.

\section{Le recensement des conflits d'usage dans la presse quotidienne régionale, méthode et composition du corpus d'analyse}

\section{MÉTHOde DE RECENSEMENT}

Afin d'observer et de rendre compte de la diversité des conflits sur l'ensemble de l'̂̂le-de-France, nous en avons fait le recensement à partir des articles rédactionnels du quotidien régional Le Parisien. La PQR (Presse Quotidienne Régionale) est le deuxième média le plus diffusé après la télévision et elle se trouve généralement en situation de quasi-monopole sur son territoire de diffusion. C'est donc en grande partie par ce biais qu'est publicisée et partagée l'actualité locale. La fine granularité temporelle du traitement des événements, sur la base d'une parution quoti- 
dienne, en fait aussi une source qui peut s'avérer très riche dans des situations nécessitant un suivi journalier ou hebdomadaire. Enfin, disponible dans chacune des régions de France, et de nombreux autres pays, elle autorise une approche comparative entre des contextes géographiques et régionaux très variés. Ce dernier point, s'il n’est pas valorisé dans cette communication, centrée sur l'Île-de-France, est au cœur d'un programme de recherche sur les conflits dans les territoires ruraux et périurbains, piloté par l'équipe Proximités de l'UMR SADAPT de l'INRA5 5

Cette source présente toutefois des biais informatifs, qui rendent les résultats de son exploitation tributaires de choix d'investigation et de publication difficilement quantifiables. Les sujets publiés sont en effet déterminés par la disponibilité et les réseaux de veille des journalistes, mais aussi par la ligne éditoriale de chaque journal (Torre et Lefranc, 2006). Plusieurs résultats de recherche ont cependant confirmé l'utilité de ce matériau sur des objets d'étude similaires, comme les activités de protestations collectives (Rucht et al., 1999).

La sélection des articles relevant de notre corpus d'étude s'est faite sur la base de critères permettant de différencier les situations de simple tension d'authentiques phases conflictuelles.

La majorité des auteurs part en effet du constat que la grande diversité des tensions liées à la multitude des usages de l'espace rend le recensement de ces dernières trop tributaire de la sensibilité de l'observateur; comme elles ne s'expriment pas toujours, un état des lieux se fondera pour partie sur des méthodes interprétatives difficilement justifiables. Les analyses empruntant à la théorie des jeux proposent alors de mobiliser la notion d'engagement crédible, pour distinguer les conflits des cas de tensions (Caron et Torre, 2005). L'engagement se décline selon des modalités plus ou moins institutionnalisées (prise de parole, signaux écrits, lettres recommandées, procédures administratives...) et plus ou moins radicales (voie de fait, panneaux d'interdiction, barrière...). Il constitue une contrainte que les acteurs s'imposent et qui engage leur positionnement dans l'avenir. Pour être crédible, cet engagement nécessite un investissement de nature monétaire ou plus hédonique.

À partir des informations issues des articles rédactionnels des pages nationales et départementales des sept cahiers franciliens (à l'exception du cahier de Paris) ${ }^{6}$ sur une année (2005), nous avons donc sélectionné les conflits de notre corpus d'étude en nous basant sur l'existence d'un acte d'engagement crédible d'au moins un acteur, visant à s'opposer à un ou plusieurs usages de l'espace périurbain (tout espace localisé au sein de la couronne périurbaine telle que définie par la classification INSEE 99 des communes).

Un conflit est décrit par un ou plusieurs articles relatant l'ensemble des événements interdépendants d'un même processus d'opposition. Pour chacun de ces processus, les informations relatives à l'objet du conflit, aux acteurs et à leurs actions, et enfin à sa localisation sont intégrées au sein d'une base de données relationnelle dédiée.

\section{LES LIMITES DE LA MÉTHODE}

En dehors des limites liées à l'exploitation d'une source indirecte que nous avons évoquées plus haut, la principale limite de cette méthode est la lourdeur du travail de dépouillement et de remplissage de la base de données relationnelle. L'exhaustivité recherchée nécessite en effet la consultation de l'ensemble des articles de chaque édition journalière afin de sélectionner les informations d'intérêt. Lorsque les archives ont été numérisées et sont consultables via un serveur de données, comme dans le cas du journal Le Parisien ${ }^{7}$, l'ergonomie de l'interface de visualisa-

5. Le recensement des conflits a été effectué sur six territoires français, caractéristiques de la diversité des contextes climatiques, géographiques et d'occupation humaine du territoire national.

6. Soit : Seine-et-Marne (77), Yvelines (78), Essonne (91), Hauts-de-Seine (92), Seine-Saint-Denis (93), Val-de-Marne (94) et Val-d'Oise (95).

7. Le service des archives du journal Le Parisien a été supprimé. Les seules archives référencées de l'ensemble des cahiers départementaux du Parisien en format papier sont à la Bibliothèque Nationale de France, mais celles-ci ne sont pas 
tion des articles est généralement dédiée à l'organisation de recherches par mots-clefs, ce qui ne correspond à pas à notre méthode. Ainsi, même si les opérations de dématérialisation des articles sont facilitées (enregistrement numérique et impression directement à partir d'un serveur accessible en ligne), la consultation exhaustive de chaque article dans une fenêtre de visualisation indépendante s'avère plus longue et moins confortable que de parcourir en une fois les doubles pages des supports papier. Au regard des moyens humains disponibles, dans le temps dédié à cette opération, ce dépouillement n'a été réalisé que pour l'année 2005 et n'a pas pu être étendu à une plus longue période. La taille du corpus doit donc amener à discuter et mettre en perspective les résultats que nous présentons ici, qui restent cependant précieux pour illustrer la problématique de la différenciation territoriale des processus conflictuels.

\section{RÉSULTATS - DESCRIPTION SYNTHÉTIQUE DU CORPUS}

Le recensement effectué selon la méthode exposée ci-dessus nous a permis de saisir dans une base de données relationnelle les informations relatives à 80 processus conflictuels, qui touchent environ 300 communes, réparties au sein de la couronne périurbaine francilienne.

La quantité d'informations pour comprendre et décrire un conflit est très variable : certaines situations n'étant relatées que par un court article, d'autres, plus durables, faisant l'objet de nombreux développements, sur plusieurs mois. Pour tous les cas, nous avons, au minimum, pu identifier l'usage contesté et le mode d'action employé pour s'y opposer. Lorsque cela était possible (la très grande majorité des cas), nous avons aussi caractérisé les acteurs, contestataires et contestés, les argumentaires développés et les enjeux protégés (Lascoumes, 1994) par les différentes parties, ainsi que la localisation des communes concernées par les usages contestés.

La grande majorité des conflits est liée au fonctionnement et à l'extension de la ville, de ses services et de ses industries (tableau 2). Parmi cette catégorie, la question des transports est omniprésente, qu'ils soient aériens (aéroports internationaux), ferrés (station TGV) ou routiers (réseau des voies de grande vitesse et des déviations locales). L'aménagement, l'exploitation et la protection des ressources naturelles fait aussi l'objet de conflits d'usage, entre partisans d'une ouverture des espaces ouverts au public (stationnement caravanes, rave party), supporters d'une valorisation économique des ressources naturelles (domaine de chasse, agriculteurs, golfs, parcs de loisir) et, bien sûr, les protecteurs de la valeur patrimoniale de ces ressources.

Ces premiers résultats nous renseignent sur les principaux processus conflictuels entre acteurs au sein de la zone périurbaine francilienne. Les argumentaires mobilisés par les contestataires s'articulent autour de deux problématiques spatiales : la problématique de la compétition entre usagers pour l'occupation de l'espace dans un territoire soumis à une forte concentration de population et d'activité; et la question des répercussions environnementales de ces objets sur les ressources territoriales (altération du paysage, contamination et dégradation du milieu, nuisances sonores).

\section{LOCALISATION DES CONFLITS}

À partir de l'exploitation de la base de données sous un Système d'Information Géographique, il est possible de localiser chaque conflit en fonction de la ou des communes où se situe l'objet de conflit. La carte ci-dessous (fig. 3) représente la répartition de ces objets au sein de l'espace régional, à partir de laquelle nous basons notre analyse des caractéristiques territoriales des processus conflictuels.

accessibles en libre consultation et doivent faire l'objet d'une demande motivée et évaluée par les conservateurs. La Bibliothèque Publique d'Information du centre Georges-Pompidou à Paris, propose la consultation en ligne des archives numérisées des principaux journaux nationaux et régionaux, dont l'ensemble des éditions d’île-de-France du Parisien, par l'éditeur Europresse [www.europresse.com], depuis les postes de la bibliothèque, mais a suspendu l'archivage des supports sous format papier ou microfilm. 


\begin{tabular}{|l|c|}
\hline Objets à la source du conflit & Nb conflits \\
\hline Infrastructures transport (route 11, aérien 4, fer 1) & $\mathbf{1 6}$ \\
\hline Infrastructures production \& transport d'énergie (dont éoliennes) & 4 \\
\hline Infrastructures services publics (scolaire, prison) & 3 \\
\hline Sites traitement des déchets (décharges, incinérateurs) & 6 \\
\hline Sites extraction (carrière, forage) & 4 \\
\hline Constructions logements/zones activités (immeubles, maisons, entrepôts) & $\mathbf{1 3}$ \\
\hline Plans d'Occupation des sols / Plans Locaux d'Urbanisme & 3 \\
\hline Stationnements caravanes & 8 \\
\hline Périmètres protection Nature & 5 \\
\hline Aménagement et utilisation des espaces ouverts (aménagement sites, loisirs) & $\mathbf{1 0}$ \\
\hline Agriculture & 4 \\
\hline Autres & 4 \\
\hline Total & 80 \\
\hline
\end{tabular}

Tableau 2 : Les objets des conflits d'usage recensés dans Le Parisien en 2005 Conflicting objects found in Le Parisien, 2005

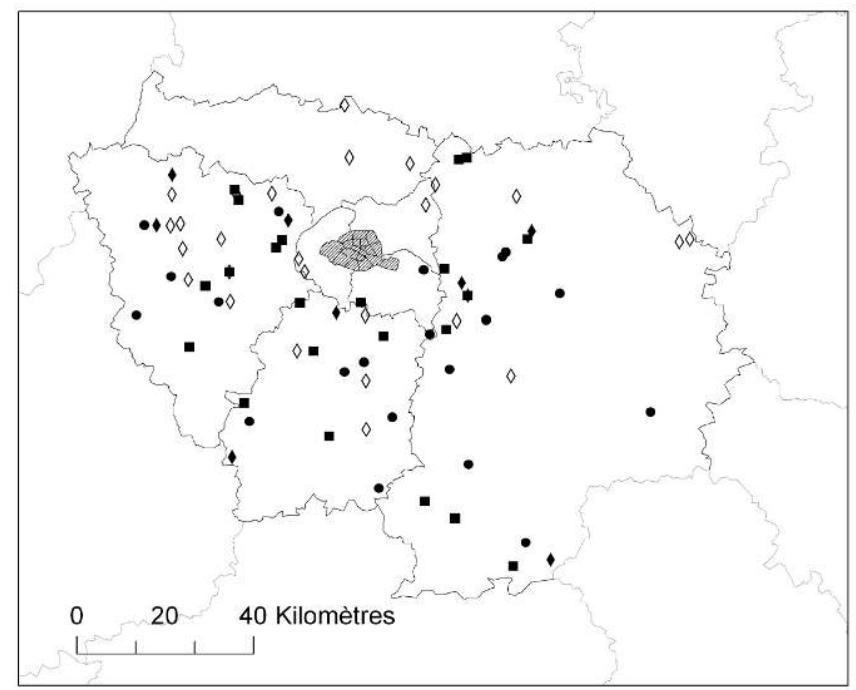

$\diamond$

Conflits causés par des infrastructures (aéroports, routes, établissements publics, éoliennes)

Conflits causés par des sites industriels dangereux (déchets, carrières)

Conflits causés par l'urbanisme (POS, extension bâti, zonages patrimonialisation)

Conflits causés par des troubles de voisinage (installation caravanes, chasse, troubles)

Figure 3 : Localisation des objets de conflit recensés en 2005 dans le journal Le Parisien (Darly, 2006). Location of conflicting objects found in Le Parisien, 2005

\section{Les espaces physiques du conflit et la différenciation des enjeux territoriaux}

Le conflit est avant tout un processus social. On peut cependant décrire la matérialité des objets et des espaces qui motivent l'engagement des différents protagonistes. Les causes spatiales d'un conflit s'inscrivent au sein de deux types d'espaces : l'espace des objets et des activités contestés, 
et l'espace des ressources protégées. Le premier nous révèle la nature des usages et activités rejetés, alors que le second nous révèle les enjeux territoriaux en matière de gestion et de partage des ressources.

\section{INSCRIPTION SPATIALE DES OBJETS ET DES NUISANCES À LA SOURCE DE LA CONTESTATION}

Tel que défini par B. Charlier (1999), puis A. Lecourt (2003), l'espace support du conflit est décrit par la nature et l'emprise des objets et activités contestés, ainsi que de leurs nuisances, mesurées ou supposées.

Ces objets sont caractérisés par leur topographie d'une part (ponctuels, surfaciques, linéaires), et par leur échelle d'implantation d'autre part (infra-communale, communale, inter-communale, département, région etc.). Ils peuvent être matériels ou immatériels.

Les objets matériels constitués par les infrastructures d'utilité publique, les aménagements liés à la circulation (aériens et routiers), au stationnement (stationnement de caravanes) et aux logements sont à l'origine d'une majorité des conflits relevés, en nombre d'articles et en nombre de situations différentes. Ces conflits visent soit à contester l'implantation de ces objets par anticipation, soit à réclamer des mesures de protection contre les nuisances émises et constatées (tableau 3).

Le second type d'objets spatiaux particulièrement polémogènes est celui du zonage ou du plan d'urbanisme ou d'aménagement. Ces conflits visent alors à contester l'application ou la validation de périmètres réglementaires qui autoriseraient des activités non désirées, ou qui mettent en place une protection environnementale particulière.

\begin{tabular}{|c|c|c|c|c|}
\hline $\begin{array}{l}\text { Étendue de l'objet } \\
\text { Objets des conflits }\end{array}$ & \multicolumn{2}{|c|}{ Échelle de la parcelle } & $\begin{array}{l}\text { Échelle de la com- } \\
\text { mune }\end{array}$ & $\begin{array}{c}\text { Supérieure à } \\
\text { l'échelle de la com- } \\
\text { mune }\end{array}$ \\
\hline $\begin{array}{l}\text { Objets matériels } \\
\text { ( } 32 \text { conflits pré- } \\
\text { ventifs, } 28 \text { conflits } \\
\text { curatifs) }\end{array}$ & $\begin{array}{c}\text { Caravanes } \\
\text { Déchets } \\
\text { Silo } \\
\text { Aérodromes } \\
\text { Forage } \\
\text { Station essence } \\
\text { Aire de stationnement }\end{array}$ & $\begin{array}{c}\text { Logements } \\
\text { Bâtiment public } \\
\text { Brulage } \\
\text { Rave Party } \\
\text { Passage } \\
\text { Culture OGM }\end{array}$ & $\begin{array}{l}\text { Déviations } \\
\text { Routières }\end{array}$ & $\begin{array}{c}\text { Aéroports internatio- } \\
\text { naux } \\
\text { Autoroutes } \\
\text { Parc Éolien } \\
\text { Élevage de sangliers } \\
\text { Ligne ferroviaire }\end{array}$ \\
\hline $\begin{array}{c}\text { Objets immatériels } \\
\text { (6 conflits préventifs, } \\
2 \text { conflits curatifs })\end{array}$ & \multicolumn{2}{|c|}{ Zonage } & POS/PLU & $\begin{array}{l}\text { Périmètre environne- } \\
\text { mental } \\
\text { Arrêté préfectoral de } \\
\text { classement }\end{array}$ \\
\hline
\end{tabular}

Tableau 3 : Échelle de l'inscription spatiale des objets géographiques à l'origine des conflits relevés dans la PQR en 2005. Certains espaces supports des conflits sont supérieurs au périmètre d'emprise de ces objets car ils rendent compte aussi des espaces touchés par les phénomènes de diffusions de nuisances ou de pollution.

Spatial scale of the conflicting objects found in Le Parisien, 2005. Most of the spaces at stake during the conflict are located within boundaries greater than the conflicting object itself. This shows that part of these conflict spaces are concerned by pollution diffusion.

Si les objets de conflit sont en majorité aménagés et implantés à l'échelle de la parcelle, la plupart d'entre eux sont contestés pour les nuisances et les flux de matières polluantes qu'ils émettent ou qu'ils sont susceptibles de produire. Dans ces cas-là, l'espace support du conflit peut s'étendre dans un périmètre beaucoup plus vaste que celui du site incriminé, producteur de nuisances. Ainsi, l'aéroport international Charles-de-Gaule, s'il s'étend sur quelques communes, est accusé d'être à l'origine de nuisances sonores et atmosphériques, qui dégradent l'environnement de centaines de communes situées à l'intérieur du périmètre de gêne sonore.

L'atmosphère véhiculant les nuisances, sonores ou olfactives, les masses d'eau vectrices de la diffusion des polluants solubles, ainsi que les blocs de terres supports de diffusion de polluants 
telluriques, sont autant de supports qui viennent étendre le périmètre de l'inscription spatiale d'un conflit.

En exploitant les informations recueillies dans notre base de données, nous avons pu cartographier l'extension spatiale des espaces supports des conflits, en prenant en compte l'extension de l'objet du conflit, mais aussi les communes dénonçant la perception ou le risque de perception de nuisances (fig. 4). En comparant l'importance spatiale des espaces supports de conflits par rapport au nombre total de conflits concernés, on peut mettre en évidence des différences territoriales significatives (tableau 4).

Figure 4 : Localisation des objets de conflits et de l'extension des espaces enjeux des conflits (Source : Le Parisien 2005 ; Darly, 2006). Location of conflicting objects and of spaces at stake during the conflicts.

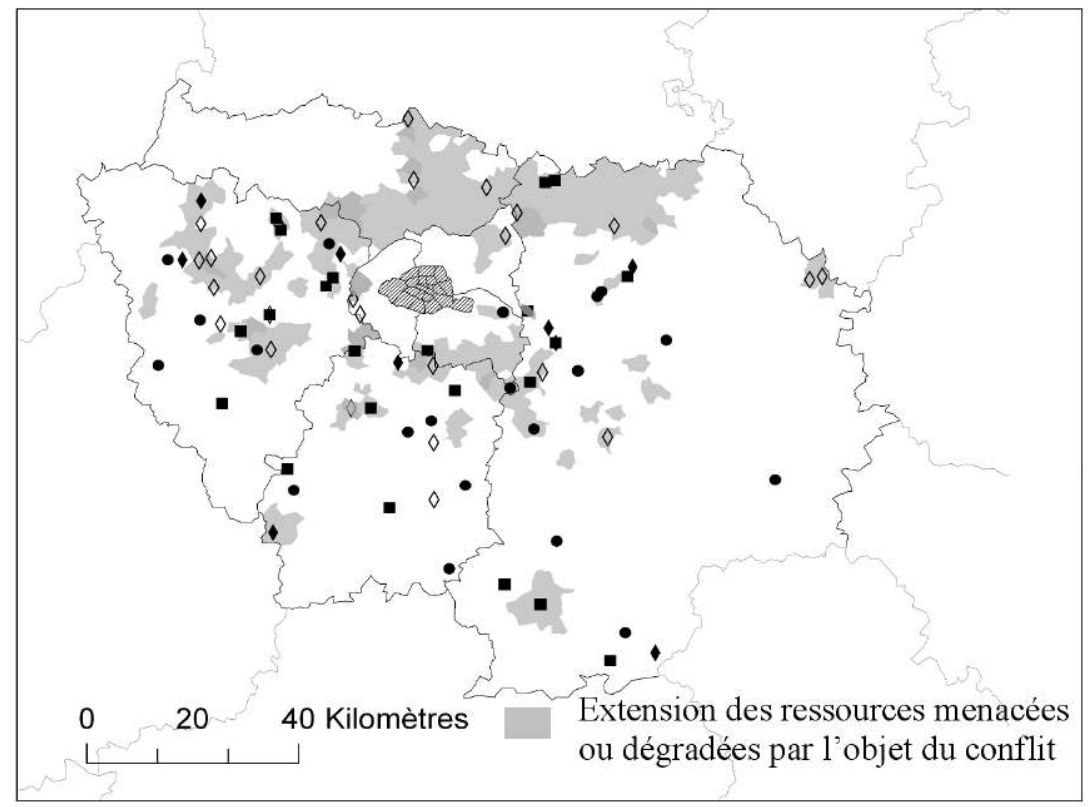

\begin{tabular}{|c|c|c|c|}
\hline & $\mathrm{Nb}$ conflits & $\begin{array}{l}\text { Extension des espaces sup- } \\
\text { ports (nb communes) }\end{array}$ & Rapport \\
\hline Ceinture de Paris & 22 & 103 & 4,7 \\
\hline Valois & 7 & 72 & 10,3 \\
\hline Brie & 23 & 52 & 2,3 \\
\hline Gâtinais & 8 & 14 & 1,8 \\
\hline Hurepoix & 10 & 14 & 1,4 \\
\hline Mantois & 22 & 61 & 2,8 \\
\hline Vexin & 5 & 10 & 2 \\
\hline
\end{tabular}

Tableau 4 : Extension des espaces supports de conflit en fonction des territoires ruraux (Source : Le Parisien, 2005)

Spreading of the spaces at stake during the conflict, depending on the rural territory concerned

Les nuisances produites par les deux aéroports internationaux d'Orly et de Roissy pèsent sur ce critère de différenciation. Elles font en effet nettement ressortir le territoire du Valois, entièrement concerné par les conflits liés à ces nuisances, ainsi que celui de la Ceinture de Paris, dont les populations du Nord-Ouest subissent les nuisances de Roissy, et les populations du Sud-Est, les 
nuisances d'Orly. Un territoire, le Mantois, est touché par des conflits relativement étendus, sans pour autant être concerné par l'aire de nuisances des aéroports internationaux. Cela peut s'expliquer par la nature des objets de conflit, parmi lesquels on retrouve des projets autoroutiers, un parc éolien de grande taille et un périmètre de classement de site étendu sur plusieurs communes.

\section{LES ENJEUX TERRITORIAUX RÉVÉLÉS PAR LES CONFLITS : ENTRE PRESERVATION ET RESTAURATION DES RESSOURCES}

En lien avec les espaces supports du conflit, le second type d'objets spatiaux à l'origine du déclenchement d'un conflit est celui des ressources territoriales, que les contestataires cherchent à préserver des nuisances et dégradations. Afin de recenser ces ressources, enjeux des conflits, nous analysons les argumentaires développés par les contestataires afin d'y identifier les « enjeux protégés» qu'ils défendent (Lascoumes, 1994). En nous basant sur l'observation empirique de nos données, nous avons traduits la nature de ces espaces protégés en termes de ressources et nous avons différencié les espaces de ressources de production (agricole ou autre), les espaces de ressources paysagères, les espaces de ressources écologiques, les espaces de ressources de consommation (résidentielle ou par des services), et enfin les espaces de ressources foncières. L'étendue et la localisation de ces espaces enjeux sont déterminées par les périmètres de l'espace support du conflit.

En mettant en regard ces dimensions de l'espace et les modes d'occupation des sols au sein de ces périmètres, le conflit devient un révélateur des enjeux sociaux attribués aux ressources localisées dans le périmètre des espaces supports du conflit.

À partir de l'observation de notre corpus de conflits (tableau 5), on constate tout d'abord que les zones les plus touchées (ou menacées) par les usages contestés sont principalement des zones d'habitat. Elles sont voisines des objets du conflit et les contestataires revendiquent que les ressources dégradées (eau, air, environnement sonore, olfactif et visuel) sont des ressources de consommation pour les particuliers qui y sont résident. Les terres agricoles sont aussi touchées par les usages contestés. Soit ces terres occupent l'emprise foncière destinée à l'aménagement envisagé, soit elles sont voisines d'une source de nuisance. Il est intéressant de constater que les contestataires revendiquent la protection de ces espaces en tant que ressources de production agricole bien sûr, mais aussi en tant que ressources paysagères et écologiques.

\begin{tabular}{|c|c|c|c|c|c|}
\hline \multirow[b]{2}{*}{$\begin{array}{l}\text { Occupation du sol des } \\
\text { périmètres touchés }\end{array}$} & \multicolumn{5}{|c|}{$\begin{array}{l}\text { Type de ressources spatiales protégées par les contestataires au sein du périmètre délimité } \\
\text { par l'objet et/ou la diffusion des nuisances contestés }\end{array}$} \\
\hline & $\begin{array}{l}\text { Ressource } \\
\text { écologique }\end{array}$ & $\begin{array}{l}\text { Ressource } \\
\text { Paysagère }\end{array}$ & $\begin{array}{c}\text { Ressources de } \\
\text { consommation } \\
\text { (cadre de vie) }\end{array}$ & $\begin{array}{l}\text { Ressources de } \\
\text { production agri- } \\
\text { cole }\end{array}$ & $\begin{array}{l}\text { Ressource } \\
\text { foncière }\end{array}$ \\
\hline Zones d'habitat (77) & - & $0,7 \%(1)$ & $39,4 \%(56)$ & - & $5,6 \%(8)$ \\
\hline Parcelles agricoles (37) & $\mathbf{5 , 6 \%} \%(8)$ & $\mathbf{5 , 6 \%} \%(8)$ & $1,4 \%(2)$ & $9,2 \%(13)$ & $\mathbf{5 , 6 \%} \%(8)$ \\
\hline $\begin{array}{l}\text { Forêt/Eau/éléments } \\
\text { naturels (28) }\end{array}$ & $9,8 \%(14)$ & $1,4 \% 2$ & $7 \%(8+2)$ & - & - \\
\hline
\end{tabular}

Tableau 5 : Enjeux liés à l'impact des usages contestés, dans le périmètre des espaces supports de conflit, en fonction de l'occupation du sol des zones touchées.

cerned.

Main type of resources impacted by the contested land use, depending on the type of space con-

Plus de 50 \% des conflits (43) sont déclenchés de manière préventive aux usages contestés, principalement dès l'annonce des projets d'aménagements, d'implantation de site sensible, ou de mise en place de périmètres de régulation.

Les conflits restants (37) sont donc déclenchés en réaction à une dégradation du milieu par l'activité urbaine (dégradation d'un site par stationnement ou construction illégale) et industrielle 
(pollution), mais aussi en réaction à certaines modalités de gestion de la nature et de régulation des usages des ressources naturelles (arrêtés préfectoraux de classement etc.). Ils ont alors une vocation curative, pour reprendre un terme médical, traduisant la volonté des acteurs locaux de restaurer les ressources territoriales dégradées.

\begin{tabular}{|l|c|c|c|}
\cline { 2 - 4 } \multicolumn{1}{c|}{} & Nb total communes & $\begin{array}{c}\text { Extension des ressources à } \\
\text { préserver par le conflit } \\
\text { (\% des communes) }\end{array}$ & $\begin{array}{c}\text { Extension des ressources à } \\
\text { restaurer par le conflit } \\
\text { (\% des communes) }\end{array}$ \\
\hline Ceinture de Paris & 221 & $\mathbf{1 5 , 8} \%$ & $\mathbf{3 0 , 8} \%$ \\
\hline Valois & 93 & $6,5 \%$ & $\mathbf{7 1 \%}$ \\
\hline Brie & $\mathbf{3 8 3}$ & $4,7 \%$ & $8,9 \%$ \\
\hline Gatinais & 138 & $5 \%$ & $\mathbf{5} \%$ \\
\hline Hurepoix & 108 & $6,5 \%$ & $4 \%$ \\
\hline Mantois & 202 & $\mathbf{2 6 , 2} \%$ & $3,5 \%$ \\
\hline Vexin & 88 & $8 \%$ & $4,5 \%$ \\
\hline
\end{tabular}

Tableau 6 : Extension spatiale des ressources à préserver et des ressources à restaurer au sein des différents territoires ruraux (Source : Le Parisien, 2005)

Spreading of spatial resources to be protected or re-establish

Là encore, les trois territoires qui se démarquent sont le territoire de la Ceinture de Paris, le territoire du Valois et celui du Mantois (Tableau 6). La localisation du premier en proximité immédiate de la première couronne parisienne explique que les ressources qui y sont localisées soient au cœur de nombreux enjeux de préservation et de restauration. Les ressources localisées dans le territoire du Valois, soumises aux nuisances de l'aéroport Charles de Gaulles font essentiellement l'objet d'enjeux de restauration par les résidents subissant ces nuisances. Dans le territoire du Mantois, c'est la reconnaissance de la valeur patrimoniale des ressources territoriales qui motive la mobilisation des acteurs contre tout usage susceptible de leur porter atteinte.

Ainsi, la nature des conflits nous révèle des territoires soumis à des dégradations environnementales prédominantes, dont la gouvernance est absorbée par les exigences de régulation des nuisances, et des territoires préservés, dont la gouvernance peut se fonder sur une affirmation des spécificités locales, réactivée au cours des processus conflictuels.

L'inscription spatiale des objets et des différents espaces, supports et enjeux, du conflit va motiver et légitimer l'engagement de certains types d'acteurs, soit parce qu'ils sont eux-mêmes usagers concurrents de ces espaces, soit parce qu'ils ont les capacités d'agir sur la régulation des usages à l'intérieur de ces périmètres. Les processus localisés vont donc faire intervenir différents groupes d'acteurs en fonction des enjeux spatiaux et de leur extension.

Les paragraphes suivants proposent de rechercher les spécificités territoriales de ces mobilisations et des figures d'opposition qu'elles suscitent.

\section{Inscription territoriale des acteurs et différenciation des figures d'opposition}

Parmi les espaces du conflit, celui des acteurs est tout aussi important que celui des objets. La géographie des acteurs impliqués dans les conflits s'est souvent focalisée sur le cas des mobilisations collectives et de l'activité des associations (Lascoumes, 1994, Charlier, 1999, et Lecourt, 2003). Les concepts de l'espace social du conflit et de l'espace de mobilisation ont alors servi d'outils de lecture et d'analyse de l'inscription spatiale des acteurs.

Dans ce cadre analytique, l'inscription spatiale d'une mobilisation, appelée espace de mobilisation, correspond bien souvent à la répartition spatiale des opposants et de leur aire d'action. Cette démarche est essentiellement liée aux caractéristiques des sources de données qui sont exploitées dans 
ces travaux. En effet, le travail de B. Charlier se base sur le dépouillement d'une revue très engagée dans la lutte écologiste, la revue Combat Nature. Dès lors, il lui permet de regarder et d'analyser les dynamiques spatiales et temporelles d'un même type de processus : l'engagement des acteurs associatifs dans l'opposition et la contestation de certains usages de l'espace naturel. De même, A. Lecourt (2003) va plus loin dans l'exploitation de cette entrée en analysant une base de données des associations de défense de l'environnement et du cadre de vie. Il caractérise l'inscription spatiale du conflit à l'aide de l'espace de référence de l'association créée. Les conflits analysés par ces auteurs sont caractérisés par une mobilisation fortement marquée par le phénomène associatif, qui se constitue soit en opposition à l'autorité publique responsable du projet, soit en substitution d'une administration environnementale faible face à des atteintes avérées au milieu naturel.

\section{PERMANENCES ET DIFFÉRENCIATION DES FIGURES D'OPPOSITION ET D'ALLIANCE ENTRE ACTEURS}

Cependant, l'analyse d'une source plus généraliste et moins dépendante des circuits d’information associatifs, comme la PQR, nous montre que d'autres acteurs interviennent de façon tout aussi prédominante que les associations environnementales (tableau 7). Ainsi, les élus et collectivités territoriales représentent à eux seuls près de la moitié des acteurs contestataires, alors que les associations en représentent un tiers.

Les acteurs contestés font intégralement partie de l'espace social du conflit, au sens de B. Charlier. Si on analyse leur composition, ce sont aussi les élus et collectivités qui sont le plus incriminés lors des conflits, alors que l'État, les industriels et les particuliers constituent le reste des groupes d'acteurs accusés.

\begin{tabular}{|l|c|c|}
\cline { 2 - 3 } \multicolumn{1}{c|}{} & Contestataires & Contestés \\
\hline Élus et collectivités territoriales & $\mathbf{4 9 \%}$ & $\mathbf{3 0 \%}$ \\
\hline Associations & $\mathbf{3 0 \%}$ & $<$ \\
\hline Particuliers & $11 \%$ & $16 \%$ \\
\hline État et agences nationales & $<$ & $21 \%$ \\
\hline Industriels & $<$ & $18 \%$ \\
\hline Total & $100 \%$ & $100 \%$ \\
\hline
\end{tabular}

Tableau 7 : Proportion des différents types d'acteurs parmi les contestataires et contestés (Source : Le Parisien 2005)

\section{Type of contested and protester actors}

Si la composition du groupe des acteurs contestés est essentiellement dépendante des objets de conflits localisés au sein des territoires ${ }^{8}$, les proportions entre les différents types d'acteurs à l'intérieur du groupe des acteurs contestataires varient relativement peu d'un territoire à un autre (Tableau 8).

On peut donc supposer que les alliances et les désaccords entre acteurs répondent à un schéma d'oppositions relativement homogène entre les territoires, et dont les spécificités locales seront essentiellement dépendantes de la nature des objets du conflit. Pour mettre en évidence la spatialité de ces schémas d'oppositions, nous proposons de porter une attention particulière à deux éléments de l'inscription spatiale d'un acteur : d'une part son territoire d'action, c'est-à-dire le périmètre au sein duquel son action est légitimée par la loi et les règlements (ou les traditions locales), et d'autre part, le territoire d'appartenance des groupes qui légitiment ses actions et ses droits d'usage, que nous appellerons son territoire de légitimation.

8. Exemples : on conteste les industriels dans le cas d'une industrie en fonctionnement, l'État et les collectivités dans le cas d'un projet d'infrastructure publique ou des particuliers dans le cas de l'installation de caravanes. 


\begin{tabular}{|c|c|c|c|c|c|c|c|c|}
\hline & \multirow{3}{*}{\begin{tabular}{c|}
$\begin{array}{c}\text { Ceinture } \\
\text { de Paris }\end{array}$ \\
$34 \%$ \\
\end{tabular}} & \multirow{3}{*}{$\begin{array}{l}\text { Valois } \\
42 \%\end{array}$} & \multirow{3}{*}{$\begin{array}{l}\text { Brie } \\
41 \% \\
\end{array}$} & \multirow{3}{*}{\begin{tabular}{c|} 
Gâtinais \\
$25 \%$ \\
\end{tabular}} & \multirow{3}{*}{\begin{tabular}{|c|} 
Hurepoix \\
$25 \%$
\end{tabular}} & \multirow{3}{*}{\begin{tabular}{|c|} 
Mantois \\
$40 \%$
\end{tabular}} & \multirow{3}{*}{$\begin{array}{l}\text { Vexin } \\
41 \%\end{array}$} \\
\hline & & & & & & & & \\
\hline \multirow{5}{*}{$\begin{array}{l}\text { Acteurs } \\
\text { contesta- } \\
\text { taires }\end{array}$} & Associations & & & & & & & \\
\hline & Collectivités & $50 \%$ & $42 \%$ & $47 \%$ & $30 \%$ & $52 \%$ & $40 \%$ & $51 \%$ \\
\hline & État & $16 \%$ & $3,5 \%$ & $2 \%$ & $6 \%$ & $2,5 \%$ & $1 \%$ & $2 \%$ \\
\hline & Particuliers & $8 \%$ & $7 \%$ & $4 \%$ & $18 \%$ & $10 \%$ & $13 \%$ & $2,5 \%$ \\
\hline & Total (+autres) & $\begin{array}{c}100 \% \\
(74)\end{array}$ & $100 \%(28)$ & $100 \%(48)$ & $100 \%(16)$ & $100 \%(40)$ & $100 \%(69)$ & $100 \%(39)$ \\
\hline \multirow{5}{*}{$\begin{array}{l}\text { Acteurs } \\
\text { contestés }\end{array}$} & $\begin{array}{l}\text { Entreprises } \\
\text { /Industries }\end{array}$ & $25 \%$ & $40 \%$ & $23 \%$ & $9 \%$ & $31 \%$ & $26 \%$ & $57 \%$ \\
\hline & Collectivités & $35 \%$ & $19 \%$ & $36 \%$ & $18 \%$ & $19 \%$ & $28,5 \%$ & - \\
\hline & État & $27,5 \%$ & $26,5 \%$ & $26 \%$ & $27 \%$ & $19 \%$ & $28,5 \%$ & $28,5 \%$ \\
\hline & Particuliers & $7,5 \%$ & - & $3 \%$ & $9 \%$ & $19 \%$ & $11 \%$ & - \\
\hline & Total (+autres) & $100 \%(40$ & $100 \%(15)$ & $100 \%(30)$ & $100 \%(11)$ & $100 \%(16)$ & $100 \%(35)$ & $100 \%(7)$ \\
\hline
\end{tabular}

Tableau 8 : Proportions des types d'acteurs contestataires ou contestés selon le territoire concerné par les conflits (Source : Le Parisien, 2005)

Type of contested and protester actors, depending on the rural territory concerned

Ainsi le territoire de légitimation d'un élu intercommunal est déterminé par son périmètre d'élection, c'est-à-dire la commune, alors que son territoire d'action se situe bien à l'échelle de l'intercommunalité. À l'inverse, l'action d'un agent de la direction départementale de l'agriculture est légitimée par le pouvoir national, alors que son action n'est légitime qu'à l'échelle du département.

\section{LES CONFLITS NOUS RÉVÈLENT LES MODALITÉS D’ARTICULATION DES ÉCHELLES DE GESTION}

Ainsi, chaque acteur peut être représenté au sein d'une grille à double entrée (fig. 5). Si on représente les principales oppositions entre acteurs au sein de cette grille, on peut mettre en évidence comment les processus conflictuels permettent d'articuler les différentes échelles de gouvernance au sein des zones périurbaines.

Le positionnement d'un acteur dans cette grille d'échelles territoriales, et le positionnement des acteurs auxquels il s'oppose, déterminent en grande partie le type d'actions qu'il peut entreprendre, ainsi que l'inscription spatiale de ces actions. Or, si la localisation et la nature des objets spatiaux est un élément essentiel de l'émergence de tensions et du déclenchement d'un conflit, ce dernier est lui-même un processus de production d'espaces et d'objets spatiaux, dont la nature et les caractéristiques sont déterminées par l'inscription spatiale des actes d'engagement et d'opposition.

Les paragraphes suivants proposent une typologie de ces productions spatiales et fournissent des éléments de connaissance sur leur distribution spatiale.

\section{Productions spatiales des conflits et registres de différenciation des espaces locaux}

Nous avons empiriquement déterminé trois registres de productions spatiales, issus des différents modes d'engagements crédibles dans le conflit que nous avons recensés. Les trois registres identifiés sont relatifs, d'une part, à l'activation de procédures de régulation et au renouvellement des espaces réglementés, d'autre part, à la publicisation des conflits et à la production d'espaces identitaires, et, enfin, à l'aménagement et à la production de nouvelles formes de l'espace physique. 
Echelle du territoire de légitimation

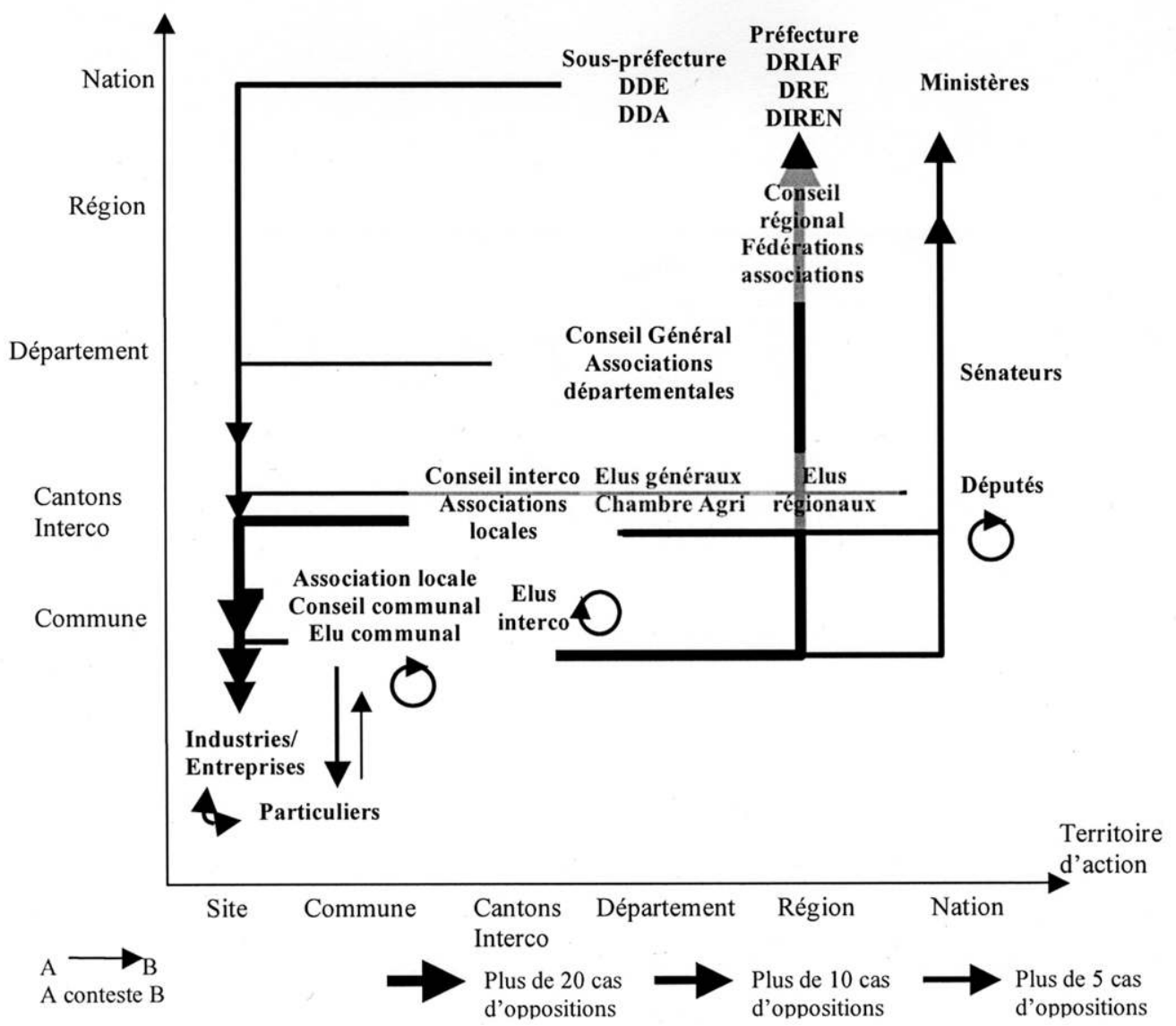

Figure 5 : Représentation schématique des interactions conflictuelles entre les différents acteurs de la gouvernance territoriale, en fonction de leurs échelles territoriales de légitimation et d'action legitimization

Conflicting relations between actors, depending on their scale of legal action, and on their scale of

\section{ACTIVATION DES PROCEDURES DE RÉGULATION ET RENOUVELLEMENT D’ESPACES RÉGLEMENTÉS}

Une grande partie des actions d'opposition se traduit par la production administrative d'avis décisionnels (arrêtés préfectoraux ou municipaux) ou consultatifs (enquête publique ou administrative), ou par le déclenchement de procédures de contentieux administratifs, judiciaires ou d'interventions policières (rédaction de procès verbaux, plaintes et recours contentieux auprès des autorités publiques, et enfin, demande d’intervention policière sur les objets contestés).

Toutes ces actions mobilisent des procédures de régulation institutionnalisées. Elles s'inscrivent soit dans le cadre d'une affirmation coercitive de la réglementation des usages de l'espace (les maires peuvent demander l'intervention des forces de l'ordre pour faire expulser des usagers illégaux sur son territoire et l'État peut ordonner la démolition d'une maison d'habitat si elle a été construite en zone inconstructible). Elles peuvent aussi s'inscrire dans le cadre d'une remise en question de la légitimité des arbitrages réglementaires qui favoriseraient certains usages de l'espace 
(les associations et collectivités n'hésitent pas à attaquer les décisions administratives en justice lorsqu'elles considèrent qu'elles vont à l'encontre de lois, décrets ou règlements qui protègent leurs propres intérêts).

Dans les deux cas, les actes d'oppositions mis en œuvre, qui caractérisent le conflit, mettent à l'épreuve l'efficacité des dispositifs de réglementations existants, à anticiper et prévenir les antagonismes d'usage au sein d'un territoire. Ils encouragent la recherche de nouveaux dispositifs de gestion, qui vont se traduire par la production de nouveaux espaces réglementés. Ainsi, les conseils municipaux peuvent prendre des arrêtés locaux pour réguler le trafic routier sur leur voirie communale. Ils peuvent aussi voter l'établissement de certaines servitudes d'urbanisme afin de prévenir l'implantation d'infrastructures urbaines. La contestation d'un périmètre environnemental peut amener à modifier les contours initialement prévus, etc.

L'ensemble de ces actions a pour conséquence de participer à la redéfinition ou à l'affirmation des espaces réglementés au sein des territoires.

\section{PUbliCISATION DES CONFLITS ET PRODUCTION D’ESPACES IDENTITAIRES}

Les actions de publicisation des conflits sont, avec le recours contentieux, les activités conflictuelles qui ont le plus attiré l'attention des chercheurs ces dernières années, notamment parce que les structures associatives, acteurs très présents lors des conflits, comme nous l'avons vu, utilisent préférentiellement et efficacement ce mode d'action. Cette publicisation se décline en fonction des supports utilisés, pétitions, démonstrations ouvertes ou diffusions écrites et électroniques. Le choix de l'échelle de visibilité des actions et de diffusion des supports se fait en fonction des espaces supports du conflit, mais aussi en fonction de l'échelle de pouvoir que les contestataires cherchent à influencer. L'activité de création d'associations locales est à ce titre un indicateur intéressant puisqu'elle implique de préciser, dans l'objet de déclaration en préfecture, le territoire d'action au sein duquel elle s'identifie.

La publicisation du conflit produit un discours et des représentations sur les espaces enjeux. Ces actions participent donc de fait du processus d'identification de nouveaux territoires, puisqu'elles concourent à le délimiter, à lui attribuer des caractères localisés et à affirmer son appropriation par un groupe d'acteurs en particulier.

On voit ainsi apparaître de nouveaux territoires de mobilisation, qui font émerger de nouveaux objets identitaires. Un exemple de ce phénomène est celui de la contestation du projet de l'autoroute A12, dont un des tracés alternatifs devait pénétrer le territoire du Parc Naturel Régional de Chevreuse, et positionner ses emprises sur un des plateaux céréaliers. Ces terres de grandes cultures, jusqu'ici peu associées à l'image du parc, ont fait l'objet d'une campagne de photographies par Yann Arthus Bertrand, résident local. Les clichés, reproduits sur des cartes postales destinées à faire pression sur les décideurs politiques, mettaient clairement en valeur les champs cultivés et la vocation de production à grande échelle. Cette étape de la campagne de contestation du tracé de l'A12 a ainsi inscrit le plateau céréalier parmi les objets de l’identité du territoire de la Haute Vallée de Chevreuse, historiquement plus connu en région parisienne pour son patrimoine forestier.

\section{AMÉNAGEMENT ET PRODUCTION DE FORMES PHYSIQUE INSCRITES DANS L'ESPACE}

Afin de s'opposer à un usage ou une activité, un des moyens les plus immédiats est d'agir directement sur la morphologie de l'espace. Ainsi, les conflits d'accès donnent régulièrement lieu à l'édification de barrières ou à la multiplication des signaux matérialisant le périmètre d'une propriété (Michel, 2003). Dans la liste des conflits que nous avons recensés, les actions sur l'espace physique sont peu nombreuses mais assez significatives pour justifier la description de ce registre. On distingue, les actions spécifiquement dédiées à la régulation de la circulation, les actions de réaménagement et d'urbanisme de zones et enfin les actions de dégradations de biens et de matériels, qui laissent des traces visibles des confrontations entre acteurs. Ces trois types 
d'action, pour s'opposer à un usage du sol, vont produire des formes, durables ou temporaires, dans le paysage. On citera comme exemple la formation de buttes de terres et de fossés aux abords des champs de jachère, qui ont pour objectif d'empêcher l'installation de caravanes lors des périodes de grands pèlerinages religieux.

\section{DiFFÉRENCIATION DES ESPACES LOCAUX}

Un même conflit est généralement caractérisé par plusieurs actes d'engagement et participe donc à plusieurs processus de production spatiale. Ces informations ont été saisies au sein de la base de données relationnelle, et permettent de mettre en évidence trois situations territoriales (tableau 9).

D'une part, les territoires au sein desquels les actions conflictuelles susceptibles de justifier la production d'espaces réglementés sont proportionnellement plus nombreuses que les autres. Il s'agit des territoires de la Brie et du Gâtinais. D'autre part, les territoires au sein desquels c'est la proportion des actions conflictuelles participant à la production d'espaces identitaires qui est plus importante. Il s'agit des territoires de la Ceinture de Paris et du Valois. Et enfin, les territoires où ces deux types d'actions sont en proportions équivalentes. Il s'agit des territoires du Hurepoix, du Mantois et du Vexin.

\begin{tabular}{|l|c|c|c|c|}
\hline $\begin{array}{c}\text { Types d'actions enga- } \\
\text { gées par les acteurs } \\
\text { contestataires }\end{array}$ & $\begin{array}{c}\text { Procédures de } \\
\text { régulation et renou- } \\
\text { vellement d'espaces } \\
\text { réglementés }\end{array}$ & $\begin{array}{c}\text { Publicisation des } \\
\text { conflits et production } \\
\text { d'espaces identitaires }\end{array}$ & $\begin{array}{c}\text { Aménagement et } \\
\text { production de formes } \\
\text { physiques }\end{array}$ & tot. conf. \\
\hline Brie & 29 & 18 & 5 & 23 \\
\hline Ceinture de Paris & 17 & 25 & 0 & 8 \\
\hline Gâtinais & 8 & 1 & 2 & 10 \\
\hline Hurepoix & 13 & 12 & 3 & 22 \\
\hline Mantois & 5 & 16 & 1 & 7 \\
\hline Valois & 5 & 3 & 0 & 7 \\
\hline Vexin & 17 & 10 & 2 \\
\hline
\end{tabular}

Tableau 9 : Proportions des différents types d'actes conflictuels au sein des différents territoires ruraux. (Source : Le Parisien, 2005)

Types of conflicting acts, depending on the rural territory concerned

Une fois encore, les territoires de la Ceinture de Paris et du Valois sont marqués par les deux processus conflictuels liés aux deux aéroports internationaux. Ces processus sont en effet caractérisés par une importante publicisation (couverture par la presse, manifestations, pétitions) qui accompagne les actes de régulation (plainte, contentieux) et cherche ainsi à influencer les décisions ministérielles dont dépendent les activités aériennes.

\section{Conclusion}

L'observation empirique des conflits à travers l'exploitation d'une source de données "généraliste » nous a permis de mettre en évidence une différenciation des processus conflictuels entre les territoires périurbains en Île-de-France. À partir de l'analyse de l'inscription spatiale des antagonismes d'usage, de l'appartenance territoriale des acteurs en opposition et des types d'actions qu'ils mettent en œuvre lors de conflits, on peut décrire plusieurs figures territoriales de la conflictualité.

La première figure est celle des territoires sous forte pression environnementale. Les conflits y sont essentiellement curatifs et les ressources déjà dégradées par les objets de conflits. Les contesta- 
taires, peu convaincus de l'efficacité des procédures de régulation, publicisent le conflit afin de sensibiliser l'opinion publique et les décideurs politiques. Les territoires de la Ceinture de Paris, du Valois et d'une partie de la Brie, sont particulièrement caractéristiques de cette figure de la conflictualité, notamment en raison de l'importance des nuisances générées par les deux aéroports internationaux d'Orly et de Roisssy. Ces territoires sont les lieux de création de nouvelles associations, principalement à l'échelle intercommunale, qui animent des dynamiques de reconstruction territoriale.

La seconde figure est celle des territoires à forte résistance identitaire. Les conflits y sont principalement préventifs et les actions de publicisation du conflit accompagnent la mise en ouvre de procédures de régulation qui interviennent en amont des projets d'infrastructures ou de réglementation. Ces processus renforcent un sentiment identitaire attaché au territoire et à ses particularités locales. Les territoires du Mantois et du Vexin (qui correspondent pour partie aux départements des Yvelines et du Val d'Oise) constituent les archétypes de cette figure territoriale de conflictualité.

Enfin, la troisième et dernière figure est celle des territoires de conflictualité occasionnelle. Les conflits y sont autant préventifs que curatifs, de faible extension spatiale, et font intervenir des procédures de régulation spécifiques aux objets du conflit (expulsion pour les campements de caravanes, recours contentieux pour l'urbanisme etc.). Les processus conflictuels localisés au sein des territoires du Hurepoix, du Gâtinais, et d'une partie de la Brie répondent en grande partie à ces caractéristiques.

Ainsi, l'analyse des conflits par la méthode de recensement des articles de presse nous a permis de mettre en évidence les spécificités territoriales des processus conflictuels. Elle constitue, à ce titre, un outil de connaissance des modalités de la gouvernance territoriale des zones périurbaines. Une extension du recensement à plusieurs années reste cependant nécessaire afin de consolider les résultats présentés dans cet article.

\section{Bibliographie}

Bertrand (N.), Tolron (J.-J.), 2002. - « Territorialisation des politiques et périurbain », Les Cahiers de la multifonctionnalité, $\mathrm{n}^{\circ} 1$, p. 85-96.

Beuret (J.-E.), 2006. - La conduite de la concertation, Pour la gestion de l'environnement et le partage des ressources, Paris, L'Harmattan, $342 \mathrm{p}$.

BRYANT (C. R.), 1995. - « The role of local actors in transforming the urban fringe ", Journal of Rural Studies, vol. $11, n^{\circ} 3$, p. 255-267.

CADÈnE (P.), 1990. - « L'usage des espaces péri-urbains. Une géographie régionale des conflits », Economie Rurale, $\mathrm{n}^{\circ} 118-119$, p. 235-267.

CADORET (A.), 2006. - Conflits d'usage liés à l'environnement et réseaux sociaux, enjeux d'une gestion intégrée? Le cas du littoral du Languedoc Roussillon, Thèse de doctorat de Géographie, Université Montpellier 3, Montpellier, $585 \mathrm{p}$.

Caron (A.), Torre (A.), 2005. - « Conflits d'usage et de voisinage dans les espaces ruraux », dans Torre (A.) et FILIPI (M.) (dir.), Proximités et changements socio-économiques dans les mondes ruraux, Paris, INRA Éditions, $322 \mathrm{p}$.

Charlier (B.), 1999. - La défense de l'environnement : entre espace et territoire, géographie des conflits environnementaux déclenchés en France depuis 1974, Thèse de doctorat de géographie, Université de Pau et des Pays de l'Adour, $750 \mathrm{p}$.

DARLY (S.), 2008. - « Tensions et conflits d'usage liés à l'agriculture. Géographie de deux corpus d'observation au sein de la région Île-de-France. » dans Loudiy (S.), Bryant (C.-R.), LAurens (L.) (dir.), Territoires périurbains et gouvernance. Perspectives de recherche, Université de Montréal, p. 109-117. 
Donadieu (P.), Fleury (A.), 2003. - « La construction contemporaine de la ville campagne », Revue de Géographie Alpine, n 4, p. 19-29.

DZIEDZICKI (J.-M.), 2001. - Gestion des conflits d'aménagement de l'espace : quelle place pour les processus de médiation?, Thèse pour le doctorat d'aménagement de l'espace et urbanisme, Tours, Université de Tours, 443 p.

Faburel (G.), 2001. - Le bruit des avions, évaluation du cô̂t social, Paris, Presses de l'école nationale des ponts et chaussées, $350 \mathrm{p}$.

GuERIN (M.), 2005. - Conflits d'usage à l'horizon 2020. Quels nouveaux rôles pour l'État dans les espaces ruraux et périurbains?, Paris, Commissariat Général du Plan, 204 p.

IAURIF, 2004. - Atlas rural et agricole de l'île-de-France, Paris, Iaurif-Driaf, 173 p.

JAiLLET (M.-C.), 2004. - «L'espace périurbain : un univers pour les classes moyennes », Esprit, n 303, p. $40-64$.

Joerin (F.), Pelletier (M.), Trudelle (C.), Villeneuve (P.), 2005 - - « Analyse spatiale des conflits urbains, Enjeux et contextes dans la région de Québec », Cahiers de Géographie du Québec, Numéro thématique Conflits, Proximité, Coopération, Vol. 49, n 138, décembre 2005, p. 319-342.

Kirat (T.) et Torre (A.), (dir.), 2006. - « Conflits d'usage et dynamiques spatiales : les antagonismes dans l'occupation des espaces périurbains et ruraux, Revue Géographie », Économie et Société, vol. 8, n 3, juillet-septembre 2006, p. 299-318.

Lascoumes (P.), 1994. - « Formes et conditions de l'action collectives », dans Lascoumes (P.), L'EcoPouvoir, environnements et politiques, La Découverte, Paris, p. 226-264.

LECOURT (A.), 2003. - Les conflits d'aménagement : analyse théorique et pratique à partir du cas breton, Thèse de doctorat de géographie, Université Rennes 2, 361 p.

Melé (P.), 2004. - «Introduction : conflits, territoires et actions publique », dans Melé (P.), LARRue (C.) et Rosemberg (M.) (coord.), Conflits et territoires, Presses Universitaires François-Rabelais, collection «Villes et territoires; 6 », p 13 à 32.

Michel (C.), 2003. - L'accès public aux espaces naturels, agricoles et forestiers et l'exercice du droit de propriété: des équilibres à gérer, Thèse de doctorat en sciences de l'environnement, ENGREF, 511 p.

PAPy (F.), TorRe (A.), 2002. - «Quelles organisations territoriales pour concilier production agricole et gestion des ressources naturelles », Études et Recherches sur les Systèmes Agraires et le Développement, $n^{\circ} 33$, p. 151-170.

Reynaud (J.-D.), 1997 - Les Règles du jeu : l'action collective et la régulation sociale, Paris, Armand Colin, $306 \mathrm{p}$.

Rucht (D.), Neidhardt (F.), 1999. - "Methodological Issues Collecting Protest Event Data : Units of Analysis, Sources and Sampling, Coding problems”, dans Rucht (D.), Koopmans (R.), Neidhardt (F.), (ed.), Act of Dissent, New Devlopments in the Study of Protest, Lanham, Rowman \& Littlefield, p. 65-89.

Torre (A.), Lefranc (C.), 2006. - « Les Conflits dans les zones rurales et périurbaines. Premières analyses de la Presse Quotidienne Régionale », Espaces et Sociétés, vol. 124-125, n 1-2, p. 93-110.

Touraine (A.), 1993. - La voix et le regard. Sociologie des mouvements sociaux, Paris, Seuil, 309 p.

Cet article a été reçu le 10 juin 2008 et définitivement accepté le $1^{\text {er }}$ décembre 2008. 